\title{
Role of weakest links and system-size scaling in multiscale modeling of stochastic plasticity
}

\author{
Péter Dusán Ispánovity, ${ }^{1, *}$ Dániel Tüzes, ${ }^{1,2}$ Péter Szabó, ${ }^{1}$ Michael Zaiser, ${ }^{2}$ and István Groma ${ }^{1}$ \\ ${ }^{1}$ Department of Materials Physics, Eötvös University, Pázmány Péter sétány 1/a, H-1117 Budapest, Hungary \\ ${ }^{2}$ Department of Materials Science and Engineering, WW8 (Materials Simulation), \\ Friedrich-Alexander-University Erlangen-Nürnberg, Dr-Mack-Str. 77, D-90762 Fürth, Germany \\ (Received 5 September 2016; revised manuscript received 4 December 2016; published 9 February 2017)
}

\begin{abstract}
Plastic deformation of crystalline and amorphous matter often involves intermittent local strain burst events. To understand the physical background of the phenomenon a minimal stochastic mesoscopic model was introduced, where details of the microstructure evolution are statistically represented in terms of a fluctuating local yield threshold. In the present paper we propose a method for determining the corresponding yield stress distribution for the case of crystal plasticity from lower scale discrete dislocation dynamics simulations which we combine with weakest link arguments. The success of scale linking is demonstrated by comparing stress-strain curves obtained from the resulting mesoscopic and the underlying discrete dislocation models in the microplastic regime. As shown by various scaling relations they are statistically equivalent and behave identically in the thermodynamic limit. The proposed technique is expected to be applicable to different microstructures and also to amorphous materials.
\end{abstract}

DOI: 10.1103/PhysRevB.95.054108

\section{INTRODUCTION}

Crystal plasticity involves important features on multiple spatial and temporal scales ranging from atomistic processes through the scales of the defect and grain microstructure up to the specimen scale. Understanding and modeling such a rich phenomenology requires multiscale approaches where different level models rely on inputs from lower scales. One aspect that has been intensively studied is the transition from discrete dislocation plasticity towards a higher scale continuum description [1-6]. The main motivation behind the quest for a continuum representation of dislocation plasticity results from the fact that dislocations interact via longrange stress fields, so in a discrete model all mutual pair interactions between dislocations have to be taken into account leading to a computational cost that makes calculations unaffordable even for systems containing comparatively few dislocations/dislocation segments. This restriction might be lifted by appropriate continuum models, in which dislocations are represented by continuous density fields, and one considers the dynamics of these fields in the form of continuity equations.

Continuum descriptions of plastic flow filter out spatial and temporal fluctuations which appear in the form of intermittent strain bursts caused by dislocation avalanches [7,8]. Such fluctuations, however, often represent important physics that one may intend to take into account. For instance, in the case of micron-scale specimens, stochastic strain bursts may interfere with predictions of formability and thus represent a major challenge for material design [9]. They also play an important role in size effects, that is, the increase of material strength if specimen dimensions are reduced down to the micron regime or below [10-12]. Thus it may be desirable to extend continuum dislocation models by a stochastic component to account for strain burst/dislocation avalanche dynamics.

\footnotetext{
*ispanovity@metal.elte.hu
}

Such a stochastic crystal plasticity model (SCPM) was proposed by Zaiser et al. in two dimensions (2D), who extended the continuum models by adding a random component to the local yield stress of the material [13]. This feature is meant to account for the internal-stress fluctuations associated with the formation and breaking of local, 'jammed' configurations such as dislocation dipoles or, in three dimensions, dislocation junctions. The model is in fact a cellular automaton (CA) representation of the plastic strain field evolution. The elementary event is the local slip of a cell (achieved in practice by the motion of one or a few dislocations between local equilibrium configurations) that induces a long-range internal stress redistribution, which in turn may trigger further events of a similar kind. The local yield threshold is updated after each event to account for the dislocation rearrangements that take place during plastic slip. The resulting model recovers the stochastic nature of plasticity and yields a power-law distribution for the random steps appearing on the stressstrain curves [14]. It also proved successful in modeling the quasiperiodic oscillatory behavior observed during slow deformation of micron-scale single crystalline pillars [15].

Interestingly, mesoscopic stochastic plasticity models were already introduced earlier with a different aim, namely, to study plasticity in amorphous materials where the dislocationmediated deformation mechanism is absent [16,17]. The basic assumptions are similar to those of SCPM: (i) plastic strain accumulates in local shear transformations that generate long-range internal stress redistribution and (ii) the material exhibits internal disorder represented by a fluctuating local yield stress. Recently, a version of such models which is completely equivalent to the SCPM of Zaiser et al. has become quite popular for studying avalanche phenomena in amorphous materials [18-21] - though we note that the description of the plastic strain field in terms of a scalar shear strain variable (which is natural in crystal plasticity where discrete directions of shear are imposed by the crystal lattice structure) appears rather artificial in the case of amorphous solids (where the atomic structure is statistically isotropic such that all possible directions of shear are equivalent). The reason why similar 
models may be used for crystalline and amorphous systems is that the long-range elastic stress induced by a local plastic slip event is independent of the underlying deformation mechanism and is described by the Eshelby solution of the corresponding eigenstrain problem [22]. In the amorphous model of Roux et al. [18] and the SCPM of Zaiser et al. [13] the adjustable parameters of the models are related to local properties - they concern the probabilistic distribution of the local yield stress and the magnitude (or distribution of magnitudes) of local slip events. These parameters are representing the microstructural features of the actual material, and are, of course, expected to differ between amorphous and crystalline solids.

In the present paper, we demonstrate how these parameters can be calibrated in the case of crystal plasticity. As the underlying lower-scale model we use conventional 2D discrete dislocation dynamics (DDD) models that have been studied extensively in the literature [23-27]. We consider load-controlled quasistatic plastic deformation where individual avalanches can be readily identified [28]. We focus on early stages of deformation (the microplastic regime before system-scale yielding occurs). In this regime we find that the stress value corresponding to the first avalanche follows a Weibull distribution, and the mean stress at the $i$ th avalanche represents a weakest link sequence from the same distribution. We show that this is not at variance with the occurrence of power-law distributed avalanches with large or even diverging sizes-though the divergence must be such that the system fraction involved in any single avalanche goes to zero in the large-system limit. We also provide an in-depth statistical analysis of the stress-strain curves and show by scaling relations that at not too large strains both DDD and SCPM exhibit a smooth plastic response if the system size tends to infinity. This tendency is characterized by a size exponent that, with an appropriate choice for the local strain increment in the SCPM, is identical for the different models. The so configured SCPM, thus, provides stress-strain curves statistically equivalent to those obtained by DDD in the microplastic regime.

The paper is organized as follows. Dimensionless units used in the paper are introduced in Sec. II, followed by the description of the plasticity models in Sec. III and the numerical results in Sec. IV. Section V presents a plasticity theory that correctly describes the numerical findings in the microplastic regime. The paper concludes with a Discussion and a Summary section.

\section{DIMENSIONLESS UNITS}

Infinite dislocation systems, provided that dislocation spacings are much larger than the dislocation core size, are invariant to the following re-scaling (see, e.g., Ref. [29]):

$$
\boldsymbol{r} \rightarrow \boldsymbol{r} / c, \gamma \rightarrow c \gamma, \text { and } \tau \rightarrow c \tau,
$$

where $\boldsymbol{r}, \gamma$, and $\tau$ denote the spatial coordinate, the plastic shear strain, and the shear stress, respectively, and $c>0$ is an arbitrary constant. This universal feature is a simple result of the $1 / r$ type (scale-free) decay of the dislocation stress fields. This property also means that in an infinite dislocation system the only length scale is the average dislocation spacing $\rho^{-1 / 2}$, where $\rho$ is the total dislocation density, so, naturally, this value is chosen as $c$. Nondimensional units can also be introduced for the strain $\gamma$ and stress $\tau$ :

$$
\boldsymbol{r}^{\prime}=\rho^{1 / 2} \boldsymbol{r}, \gamma^{\prime}=\gamma /\left(b \rho^{1 / 2}\right), \tau^{\prime}=\tau /\left(\frac{\mu b}{2 \pi(1-v)} \rho^{1 / 2}\right),
$$

where $b$ is the length of the Burgers vector which defines the elementary 'quantum' of displacement, $\mu$ is the shear modulus, and $v$ is the Poisson ratio. Throughout the paper these dimensionless units will be used, and the distinguishing $(\cdot)^{\prime}$ symbol will be omitted.

\section{SIMULATION METHODS}

In this paper, for simplicity, two-dimensional (2D) models are considered. All three models introduced below have been used extensively in the literature, therefore, only their main features are summarized.

\section{A. Stochastic continuum plasticity model (SCPM)}

The model is based on the crystal plasticity model introduced by Zaiser and Moretti [13] and considers a plane strain (2D) problem where slip occurs on a single slip system. The local scalar plastic shear strain field is denoted as $\gamma_{\mathrm{pl}}(\boldsymbol{r})$ and the corresponding local resolved shear stress as $\tau_{\text {loc }}(\boldsymbol{r})$. In an infinite system one can write the shear stress at an arbitrary position $\boldsymbol{r}$ as

$$
\tau_{\mathrm{loc}}(\boldsymbol{r})=\tau_{\mathrm{ext}}+\left(G_{E} * \gamma_{\mathrm{pl}}\right)(\boldsymbol{r}),
$$

i.e., it consists of two parts: an 'external' stress, understood as a homogeneous stress created by remote boundary tractions, plus an internal stress generated by the inhomogeneous $\gamma_{\mathrm{pl}}(\boldsymbol{r})$ field via $G_{E}(\mathbf{r})$, the elastic Green's function specified by the corresponding Eshelby inclusion problem [22]. In the plane strain problem considered all these fields are scalar fields and * stands for spatial convolution. The stress and strain fields are discretized on a square lattice with cell size $d$ (measured in dimensionless units introduced in Sec. II) of global size $L \cdot d \times L \cdot d$ with the edges parallel to the $x$ and $y$ direction and $L=8,16, \ldots, 512$. Accordingly space coordinates are discretized, $x=i \cdot d, y=j \cdot d$ where $i, j$ are integers. The discretized Green's function $G_{E}(i, j)$ is proportional to the stress field of a local slip at the origin, $\gamma_{\mathrm{pl}}(i, j)=\delta_{i j} \Delta \gamma_{\mathrm{pl}}$ where $\delta_{i j}=1$ if $i=j=0$ and zero otherwise. It is numerically evaluated as the stress field of four edge dislocations with Burgers vectors $b \boldsymbol{e}_{x}, b \boldsymbol{e}_{y},-b \boldsymbol{e}_{x}$, and $-b \boldsymbol{e}_{y}$ at the right, top, left, and bottom sides of the slipped cell, respectively. This corresponds to a local plastic shear of $\Delta \gamma_{\mathrm{pl}}=2 / d$. The stress values are evaluated at the center points of the cells which, for example, gives in the origin the result $G_{E}(0,0) \Delta \gamma_{\mathrm{pl}}=$ $-4 \Delta \gamma_{\mathrm{pl}}=-8 / d$. For the other cells the numerical values of $G_{E}(i, j)$ can be seen in units of $\left|G_{E}(0,0)\right|$ in Fig. 1.

Internal structural disorder is taken into account in terms of a fluctuating local threshold value $\tau_{\text {th }}$. This means that if for a given cell

$$
\tau_{r}(\boldsymbol{r}, t):=\tau_{\mathrm{th}}(\boldsymbol{r}, t)-\left|\tau_{\mathrm{loc}}(\boldsymbol{r}, t)\right|>0
$$

holds, then it is in equilibrium, otherwise it is active, that is, it yields. We assume that cells are large enough to neglect correlations between the threshold values of adjacent cells, making the thresholds independent random variables. Their 


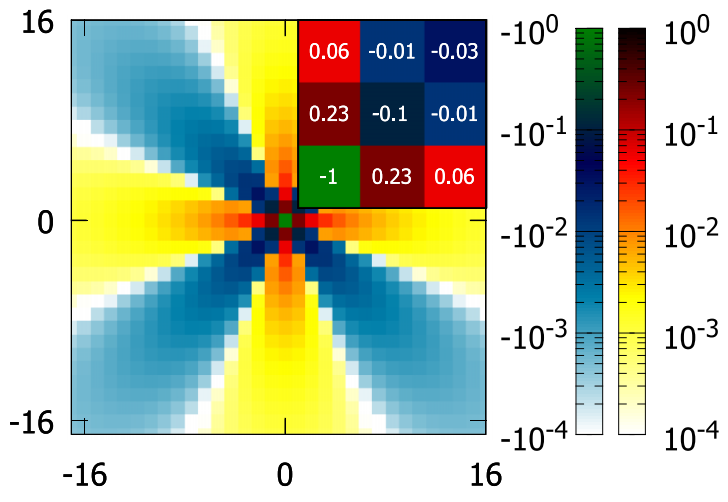

FIG. 1. The center part of the stress field of an elementary slip event $\Delta \gamma_{\mathrm{pl}}$ for the case $L=128$ in units of $\left|G_{E}(0,0)\right| \Delta \gamma_{\mathrm{pl}}$. In the upper right corner a magnification of the cells $[0,2] \times[0,2]$ is shown. Note the fourfold symmetry.

values are chosen from Weibull distributions with shape parameters $\beta=1,1.4$, and 2, and scale parameter $\tau_{w}$.

At the beginning of a simulation initial values of the local thresholds are assigned and the strain (and thus also the local stress) is set to zero everywhere. Then a stress-controlled loading procedure is implemented as follows. The external stress is increased until Eq. (4) is violated in a single cell, which then becomes active. At this cell the local strain is increased by $\Delta \gamma_{\mathrm{pl}}$ and a new local threshold value is assigned from the threshold distribution. The internal stress is recalculated according to Eq. (3) and the newly activated cells are determined using Eq. (4). As long as $\tau_{r} \leq 0$ holds for at least one cell, the system is in an avalanche, and the local strain is increased by $\Delta \gamma_{\mathrm{pl}}$ in the cell where $\tau_{r}$ is the smallest (extremal dynamics). When for all the cells $\tau_{r}>0$ holds the avalanche ceases and the external stress is further increased by the smallest $\tau_{r}$ required to trigger the next avalanche. At every state of the system the total strain is defined as the spatial average of the local strains: $\gamma:=\left\langle\gamma_{\mathrm{pl}}\right\rangle$.

\section{B. Discrete dislocation dynamics}

\section{Continuous representation (TCDDD)}

The model called time-continuous discrete dislocation dynamics (TCDDD) considered here consists of $N$ straight parallel edge dislocations, all of which belong to the same slip system. The model aims to describe the easy slip regime of fcc single crystals at low temperatures, where dislocation glide is dominant. Therefore, climb and cross-slip are neglected.

The slip direction is defined as the $x$ direction and chosen parallel to one of the sides of the square-shaped simulation area, so the Burgers vectors of the dislocations may point in two directions defining two possible signs $s$ : $\boldsymbol{b}_{i}=s_{i}(b, 0)$, where $i=1, \ldots, N$ and $s_{i} \in\{-1,1\}$. Dislocations of both signs are assumed to be present in equal amounts. Since there is only one slip direction, the glide dislocation interactions can be described in terms of the shear stress fields induced by each dislocation. Its form in the dimensionless units introduced above is $s \cdot \tau_{\text {ind }}(\boldsymbol{r})$, where $\boldsymbol{r}=(x, y)$ is the relative displacement from the dislocation,

$$
\tau_{\text {ind }}(\boldsymbol{r})=x\left(x^{2}-y^{2}\right) /\left(x^{2}+y^{2}\right)^{2}=r^{-1} \cos (\varphi) \cos (2 \varphi)
$$

is a spatially decaying anisotropic function, and $(r, \varphi)$ are the corresponding polar coordinates. To model an infinite crystal periodic boundary conditions are applied and the periodic form of Eq. (5) is used (for details see, e.g., Ref. [30]).

The system is driven by an external shear stress $\tau_{\text {ext }}$ which is considered spatially homogeneous over the region of interest, hence, the equation of motion of the $i$ th dislocation is:

$$
\dot{x}_{i}=s_{i}\left[\tau_{\mathrm{ext}}+\sum_{j=1 ; j \neq i}^{N} s_{j} \tau_{\text {ind }}\left(\boldsymbol{r}_{i}-\boldsymbol{r}_{j}\right)\right], \quad \dot{y}_{i}=0,
$$

where $\boldsymbol{r}_{i}=\left(x_{i}, y_{i}\right)$ denotes its position, and the dislocation mobility is absorbed into the timescale. Here it is assumed that due to the strong phonon drag the motion is over-damped and, thus, inertial terms can be neglected.

The simulations are started from an initially random dislocation arrangement. First, Eq. (6) is solved at $\tau_{\mathrm{ext}}=0$ until the system reaches equilibrium. Then a quasistatic load-controlled procedure is applied, i.e., the driving stress $\tau_{\text {ext }}$ is increased at a fixed rate between avalanches, and is kept constant during active periods where the strain rate exceeds a small threshold (for details see Ref. [30]). The plastic strain at time $t$ is obtained using $\gamma(t)=\sum_{i=1}^{N} s_{i}\left(x_{i}(t)-x_{i}(0)\right)$. As discussed in Ref. [31], plastic activity in this model has two different aspects: During avalanches, the rate of plastic deformation is high and quite independent on the external driving rate. In between avalanches, on the other hand, there still is dislocation motion but the corresponding strain rate is proportional to the external stress rate and the motions are quasireversible. Both regimes can be well separated by a judicious choice of the strain rate threshold used in the definition of an avalanche.

In the dimensionless units introduced above the linear system size is related to the number of dislocations via $L=N^{0.5}$. The simulations are repeated for different system sizes $L=8,11.31,16,22.63,32$ with large ensembles of statistically equivalent realizations in each case (consisting of $3000,2000,800,300$, and 180 individual runs, respectively). Very narrow dislocation dipoles are removed since they practically do not affect the dynamics but due to numerical reasons slow down the simulations considerably.

\section{Cellular automaton representation (CADDD)}

The cellular automaton discrete dislocation dynamics (CADDD) is very similar to the continuous method introduced above except for two important differences:

(1) Space is discretized, meaning that dislocations move on a regular equidistant grid, and only one dislocation may be present in a cell at the same time. In the simulations performed the cell size $\delta$ was 128 times smaller than the average dislocation spacing, meaning that only every $128 \times 128$ th cell was populated.

(2) Time is also discretized, i.e., the dynamics is defined by a rule that controls how to move dislocations from one cell to a neighbor cell. Here we use extremal dynamics (ED), meaning that the stress induced by the other dislocations $\tau$ [i.e., the RHS of Eq. (6)] is evaluated at the right (left) border of the cell containing the dislocation. If for a dislocation the force $s_{i} \tau>0(<0)$ then a step in the right (left) direction is 
energetically favorable and the decrease in the stored elastic energy $\Delta E$ is proportional to $-|\tau| \delta$. Such a dislocation is called active. In every time step the active dislocation with the highest energy drop is moved, then the interaction stresses are recomputed. If there is no dislocation eligible to move (that is, $\Delta E>0$ for each) then the external stress is increased until a dislocation starts to move. If two dislocations of opposite sign occupy the same cell, they are annihilated.

As seen, the driving is similar to the quasistatic load control of the TCDDD. The simulations are also started from a random dislocation configuration, an initial relaxation is performed, and different system sizes are considered. The advantage of this model lies in the higher computational speed compared to TCDDD that can be achieved by tabulating the dislocation interactions, which allows much larger systems to be studied. In addition, it allows us to test the role of the chosen dynamics (overdamped or extremal) for the results obtained.

There are however some issues concerning the definition of strain bursts. In the CADDD model such a burst can be easily defined in exactly the same manner as in the SCPM model - a burst is ongoing as long as at least one dislocation is active, and the burst strain of burst number $k$ is evaluated as $\gamma^{(k)}=\sum_{i=1}^{N} s_{i}\left(x_{i}(k)-x_{i}(k-1)\right)$ where $x_{i}(k)$ is the position of dislocation $i$ at the end of burst $k\left[x_{i}(0)\right.$ is the position after the initial relaxation, before the first stress increase triggers the first burst of the sequence]. Such a definition leads to an avalanche size distribution which is, in the regime of large avalanches, completely equivalent to that of the TCDDD model [28]. However, in the regime of small avalanches problems arise since the CADDD dynamics by its structure does not allow us to impose a strain rate threshold that would allow us to separate quasireversible dislocation motions in between avalanches from the strongly irreversible motions occurring, subsequent to some local stress threshold being crossed, during the avalanches themselves. Rather, the quasireversible dislocation motions are broken into sequences of small deformation steps which cannot be easily distinguished from small avalanches. This is not a major issue when it comes to macroscopic deformation, avalanche statistics, and stress-strain curves, all of which are dominated by large avalanches, but it causes problems when we want to do a statistics of avalanche initiation stresses. As far as the statistical properties of avalanche initiation stresses and their sequences are concerned, we therefore shall exclusively refer to the TCDDD model.

\section{NUMERICAL RESULTS}

In this section simulation results are provided using the plasticity models introduced in Sec. III. In every model, the obtained stress-strain curves are steplike and differ among realizations. In the following, the statistical properties of the stress-strain curves will be examined followed by an analysis of the stress and strain sequences corresponding to individual strain bursts. The latter ones (denoted by $\tau^{(k)}$ and $\gamma^{(k)}$, respectively) are defined by the sketch of Fig. 2. In the rest of this paper, for simplicity, the external stress $\tau_{\text {ext }}$ will be denoted as $\tau$.

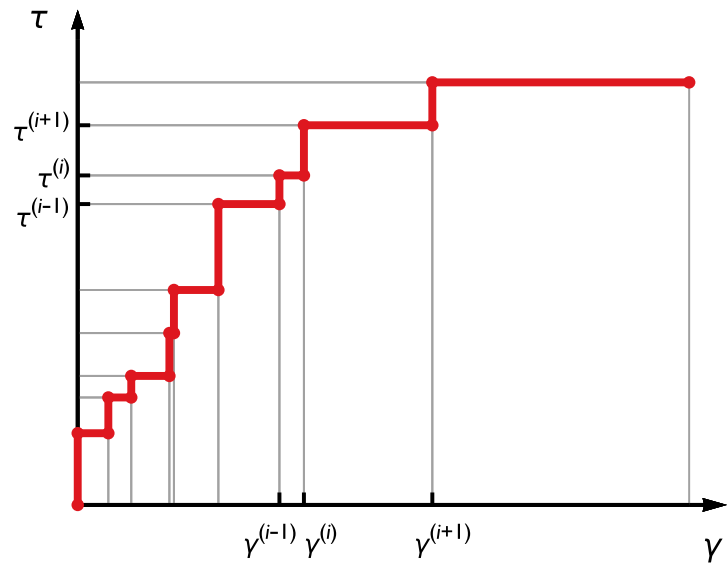

FIG. 2. Sketch of a stress-strain curve obtained by the models of Sec. III. The curve can be fully characterized by the stress and strain sequences $\tau^{(k)}$ and $\gamma^{(k)}$.

\section{A. The SCPM model}

There are three scalar parameters of the SCPM: (i) the $\beta$ shape parameter of the Weibull distribution describing the local yield stress distribution, (ii) $\Delta \gamma_{\mathrm{pl}}$ determining the local increment of the strain during the activation of a cell, and (iii) $\tau_{w}$ characterizing the average strength (threshold stress) of the cells. We note that one of the last two parameters can be always eliminated by measuring stress in units of $\tau_{w}$ and strain in units of $\Delta \gamma_{\mathrm{pl}}$, which leaves a nondimensional coupling constant $I=\Delta \gamma_{\mathrm{pl}} / \tau_{w}$ as well as the Weibull exponent $\beta$ as independent parameters. In the following section we present simulations with different parameters, and if not stated otherwise $\beta=1.4$, $\Delta \gamma_{\mathrm{pl}}=1 / 4$, and $\tau_{w}=1$ (that is, $\beta=1.4$ and $I=1 / 4$ ) are used.

\section{Average stress-strain curve}

Figure 3(a) plots the average stress-strain curves obtained by the SCPM simulations at different system sizes and for different values of the exponent $\beta$. The curves were obtained by the following method: For a given strain value $\gamma$ the assigned stress value $\langle\tau\rangle$ is the average of the stress values measured in individual simulations at $\gamma$. This procedure was repeated for different $\gamma$ values to obtain the whole average stress-strain curve. It is seen that the microplastic regime is described by a power law,

$$
\langle\tau\rangle(\gamma)=\tau_{1} \gamma^{\alpha}
$$

over several decades where $\tau_{1}$ is a constant prefactor and the exponent $\alpha$ depends on the value of the Weibull parameter $\beta$. The fitted values of $\alpha$ are summarized in Table I. It is interesting to note that the curves do not exhibit a clear sign of size effects: For a given $\beta$ the values obtained for different $L$ overlap and therefore, no $L$ dependence was included in Eq. (7). For plastic strains $\gamma \gtrsim 1$ the stress saturates and the system enters a continuously flowing state.

Figure 3(b) shows the role of the two other parameters $\Delta \gamma_{\mathrm{pl}}$ and $\tau_{w}$. As seen, the average stress-strain curves do depend on the specific choice, but according to the inset, a scaling collapse in terms of the coupling constant $I$ can be obtained if stress and strains are rescaled by $\tau_{w}$ and $\Delta \gamma_{\mathrm{pl}}$. At low stresses/strains, 

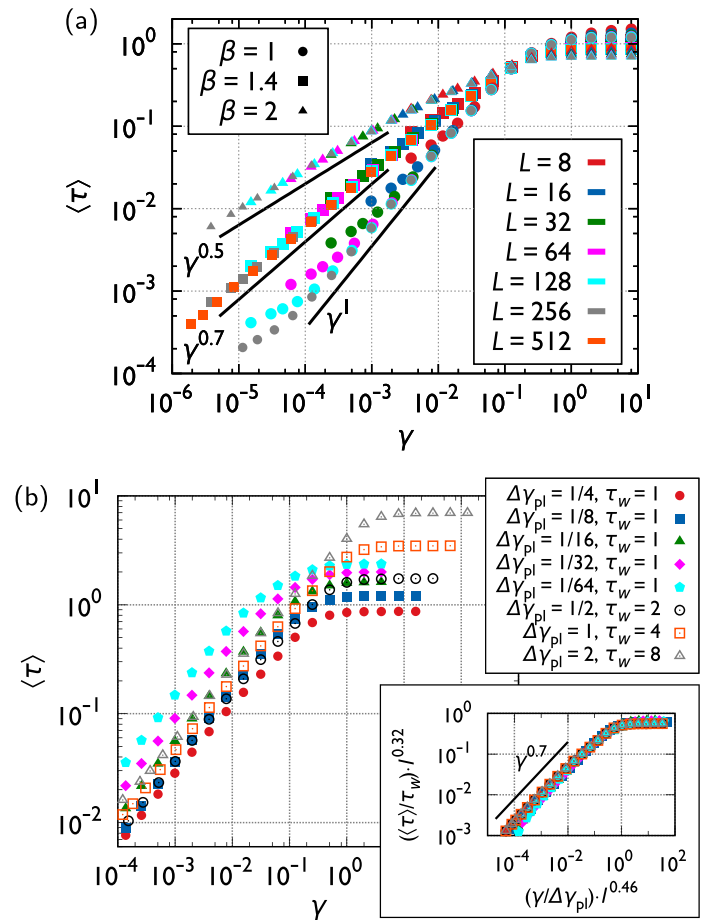

FIG. 3. Average stress-plastic strain curves obtained by the SCPM with different choices of the parameters. In every case for small strains they follow a power law, then saturate. (a) Effect of the shape parameter $\beta$. The power-law region is consistent with the $\langle\tau\rangle=\gamma^{1 / \beta \zeta}$ relation predicted by Eq. (30) with $\zeta=1$. (b) Effect of $\tau_{w}$ and $\Delta \gamma_{\mathrm{pl}}$ at $\beta=1.4$ and $L=128$. According to the scaling collapse of the inset the average stress-strain curves obey $\left(\langle\tau\rangle / \tau_{w}\right) I^{0.32}=\left(I^{0.46} \gamma / \Delta \gamma_{\mathrm{pl}}\right)^{0.7} f\left(I^{0.46} \gamma / \Delta \gamma_{\mathrm{pl}}\right)$ with a suitable function $f$ where $f(x) \rightarrow$ const. if $x \ll 1$ and $f \propto x^{-0.7}$ if $x \gg 1$.

the curves exhibit a power-law regime which only depends on the parameter $\beta$.

\section{Fluctuations in the plastic response}

Although the averaged stress-strain curves are smooth, the stress-strain curves corresponding to individual realizations are staircaselike and differ from each other. Here we investigate the cumulative distribution of stresses $\Phi_{\gamma}(\tau)$ measured at a given strain $\gamma$ for different realizations. The wider this distribution is, the larger the scatter of stress $\tau$ among the individual realizations. Macroscopic bodies are characterized by a well-defined and smooth stress-strain curve, so for large systems one expects shrinking of the distribution width. Indeed, as seen in Fig. 4 for all strains $\gamma$ the measured $\Phi_{\gamma}(\tau)$ curves tend towards step functions as the system size increases. Since there is only a negligible size effect in the average stress-strain curve, the stress-strain response of an infinite system must be equal to $\langle\tau\rangle(\gamma)$, therefore, the limiting step function must be located at $\langle\tau\rangle(\gamma)$. Interestingly, the $\Phi_{\gamma}(\tau)$ curves seem to intersect with each other in exactly this point.

According to the inset of Fig. 4 the curves can be collapsed by rescaling the stresses by the system size around $\langle\tau\rangle(\gamma)$. In addition, the curves can be fitted well by a normal distribution, that is,

$$
\Phi_{\gamma}(\tau)=\frac{1}{2}\left[1+\operatorname{erf}\left(\frac{\tau-\langle\tau\rangle(\gamma)}{c L^{-\theta}}\right)\right]
$$

The reason why the stress distribution at a given finite strain is well approximated by a Gaussian will be explained in Sec. V. In Eq. (8), $\langle\tau\rangle(\gamma)$ is the average stress at strain $\gamma$ as described by Eq. (7), $\theta=1 \pm 0.05$ is the exponent characterizing the system size dependence of the stress fluctuations, and $c$ is an appropriate constant.

We note that to fit the same distribution for $2 \mathrm{D}$ and 3D DDD as well as micropillar compression data, previously a shifted Weibull distribution has been used [32]. Here we use a Gaussian which can represent the data with only two fit parameters and besides is consistent with the theoretical arguments presented later on (Sec. V) which predict a normal distribution in the large system limit.

\section{The stress sequence}

The two following subsections aim at studying the statistics of the stress and strain sequences $\tau^{(i)}$ and $\gamma^{(i)}$, because they will play a central role in the simple plasticity model described in Sec. V. First, we consider the distribution $\Phi^{(1)}$

TABLE I. Summary of exponents used in the paper.

\begin{tabular}{|c|c|c|c|c|c|}
\hline Exponent & Description & $\begin{array}{l}\text { Value predicted } \\
\text { by theory }\end{array}$ & $\begin{array}{l}\text { Value for } \\
\text { SCPM }\end{array}$ & $\begin{array}{l}\text { Value for } \\
\text { TCDDD }\end{array}$ & $\begin{array}{l}\text { Value for } \\
\text { CADDD }\end{array}$ \\
\hline$\beta$ & $\begin{array}{l}\text { Characterizes threshold stress distribution, } \\
\text { see Eq. (15) }\end{array}$ & - & $\begin{array}{l}1.0,1.4 \text {, and } \\
2.0^{\mathrm{a}}\end{array}$ & $1.4 \pm 0.05$ & - \\
\hline$\eta$ & $\begin{array}{l}\text { Describes the relation between the system size } L \\
\text { and the number } M \text { of statistically independent } \\
\text { avalanche initiation sites, see Eq. (21) }\end{array}$ & - & $2.0 \pm 0.05$ & $1.6 \pm 0.1$ & - \\
\hline$\zeta$ & Characterizes the strain sequence, see Eq. (28) & - & $1.0 \pm 0.05$ & $0.9 \pm 0.05$ & - \\
\hline$\xi$ & $\begin{array}{l}\text { Characterizes the system size dependence of the } \\
\text { average avalanche size, see Eq. (26) }\end{array}$ & $\eta \zeta$ & $2.0 \pm 0.1$ & $1.5 \pm 0.1$ & - \\
\hline$\alpha$ & $\begin{array}{l}\text { Exponent of the power-law characterizing the } \\
\text { microplastic regime of the stress-strain curves, } \\
\text { see Eq. ( } 7)\end{array}$ & $(\beta \zeta)^{-1}$ & $\begin{array}{c}1.0 \pm 0.05,0.7 \pm 0.05, \text { and } \\
0.5 \pm 0.05\end{array}$ & $0.8 \pm 0.05$ & $0.8 \pm 0.05$ \\
\hline$\theta$ & $\begin{array}{l}\text { Exponent characterizing the system size } \\
\text { dependence of the stress fluctuations, see Eq. (8) }\end{array}$ & $\eta / 2$ & $1.0 \pm 0.05$ & $0.8 \pm 0.05$ & $0.8 \pm 0.05$ \\
\hline
\end{tabular}

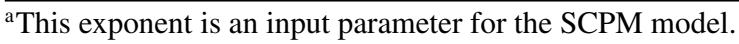



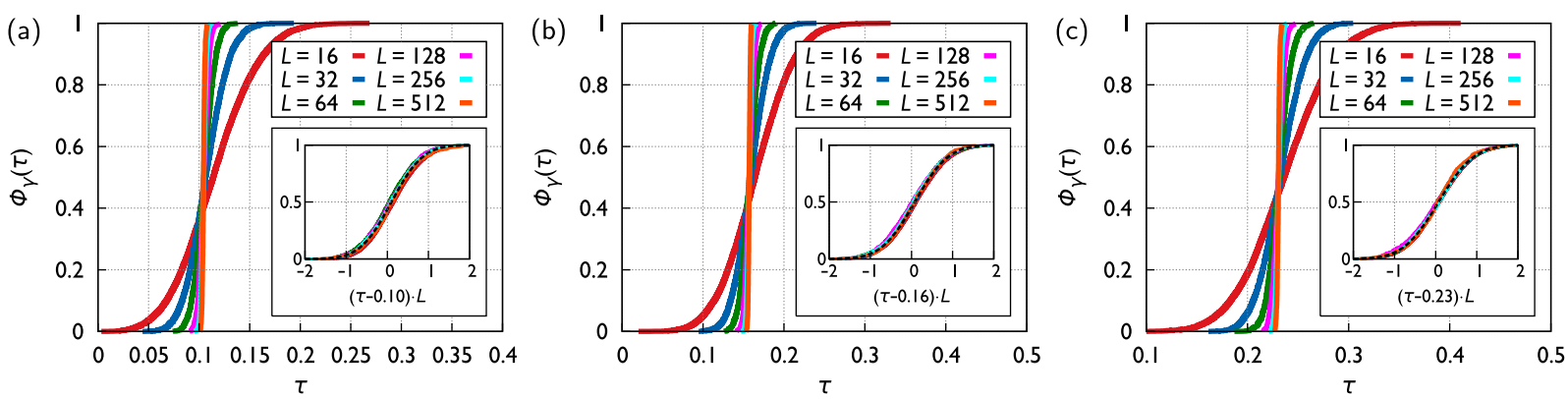

FIG. 4. Cumulative stress distributions at different deformation levels for the SCPM model. As system size increases the distributions tend to a step function, that is, stress fluctuations disappear for large samples. By multiplying the external stress with a power of the system size one can fit the curves with a normal distribution (dashed line) as shown in the insets. (a) $\gamma=0.008$, (b) $\gamma=0.016$, (c) $\gamma=0.032$.

of the stress $\tau^{(1)}$ where the first event takes place. In the SCPM model the plastic strain is initially zero everywhere, therefore, until the occurrence of the first event the local stress is equal to the applied stress in every cell. Consequently, the distribution of the initiation stress of the first plastic event must be a Weibull distribution with shape parameter $\beta$ and scale parameter proportional to $L^{-2 / \beta}$ (see Sec. V A for details). Indeed, according to Fig. 5(a) $\Phi^{(1)}$ is well represented by the corresponding Weibull distribution, and distributions pertaining to different system sizes overlap if stress values are rescaled by $L^{2 / \beta}$.

Figures 5(b) and 5(c) plot the average stress sequence $\left\langle\tau^{(i)}\right\rangle$ and its standard deviation (STD) $\delta \tau^{(i)}$, respectively. The curves corresponding to a given $\beta$ parameter overlap for small $i$ values (that is, when $\tau^{(i)} \lesssim 0.1$ ) if the stresses are rescaled by $L^{2 / \beta}$. The curves are well described by the power laws

$$
\begin{aligned}
\left\langle\tau^{(i)}\right\rangle & =\tau_{0}\left(\frac{i}{L^{\eta}}\right)^{1 / \beta}, \\
\delta \tau^{(i)} & =\frac{\tau_{0}}{i^{1 / 2}}\left(\frac{i}{L^{\eta}}\right)^{1 / \beta},
\end{aligned}
$$

with $\eta=2.0 \pm 0.05$ yielded by visual inspection.

\section{Strain sequence}

The average and the STD of the strain sequence $\gamma^{(i)}$ are seen in Fig. 6. Both $\left\langle\gamma^{(i)}\right\rangle$ and $\delta \gamma^{(i)}$ follow a power law for small $i$ values:

$$
\begin{gathered}
\left\langle\gamma^{(i)}\right\rangle=s_{0} \frac{i^{\zeta}}{L^{\xi}}, \\
\delta \gamma^{(i)}=\frac{s_{1}}{i^{1 / 2}} \frac{i^{\zeta}}{L^{\xi}},
\end{gathered}
$$

with $\zeta=1.0 \pm 0.05$ and $\xi=2.0 \pm 0.1$. It is important to note that neither $\zeta$ nor $\xi$ are sensitive to the choice of the exponent $\beta$, but the actual level of $\gamma^{(i)}$ and its scatter (and, thus, the values $s_{0}$ and $s_{1}$ ) are significantly larger for smaller $\beta$ values, which means that individual avalanches are much larger in this case.

\section{B. DDD models}

\section{Average stress-strain curve}

The average plastic response of the specimens was calculated in the same manner as for SCPM described in Sec. IV A 1.
According to Fig. 7 the average stress-strain curves show similar features to those obtained by the SCPM: (i) the microplastic regime is characterized by a power law with an exponent $\alpha=0.8 \pm 0.05$ [see Eq. (7)] and only a weak indication of size effects is seen, (ii) this regime breaks down at $\tau \approx 0.1$, and (iii) the stress-strain curves saturate for large $(\gamma \gtrsim 1)$ strains.

The only significant difference in the average stress-strain curves between different TCDDD and CADDD simulations appears at large $(\gamma \gtrsim 1)$ strains. As discussed in Ref. [27] the behavior of 2D CDD models in this regime may be strongly influenced by artifacts of the periodic boundary conditions. However, the statistical arguments presented in the following apply to the initial part of the stress-strain curve, i.e., they are valid for small to medium strains only where the two models yield quite similar behavior.

\section{Fluctuation in the plastic response}

Cumulative distributions $\Phi_{\gamma}(\tau)$ of the stresses measured for different realizations at a given strain $\gamma$ are plotted in Fig. 8 . As in the case of SCPM, they (i) tend to step functions for large systems, (ii) the curves for different system sizes intersect in a single point, (iii) by scaling the stress with a power of the system size, scaling collapse can be obtained, and (iv) the curves are well represented by normal distributions. This means that Eq. (8) is valid with an exponent $\theta=0.8 \pm 0.05$ that is somewhat smaller than the value $\theta=1$ obtained for SCPM.

\section{The stress sequence}

The following two subsections investigate the statistics of stress and strain sequences introduced above. As discussed in Sec. III B 2, these sequences cannot be unambiguously defined for CADDD, as it does not allow us to distinguish small avalanches from quasireversible dislocation motions during quasistatic loading. We, therefore, constrain ourselves to the TCDDD simulations. First, as for the SCPM, the cumulative distribution $\Phi^{(1)}$ of $\tau^{(1)}$, i.e., the stress where the first plastic event sets on, is calculated. According to Fig. 9 (a) $\Phi^{(1)}$ can be fitted perfectly by a Weibull distribution with shape parameter $\beta$ that can be collapsed for different system sizes when rescaled by $L^{\eta / \beta}$, with parameters

$$
\begin{gathered}
\beta=1.4 \pm 0.05, \\
\eta=1.6 \pm 0.1 .
\end{gathered}
$$



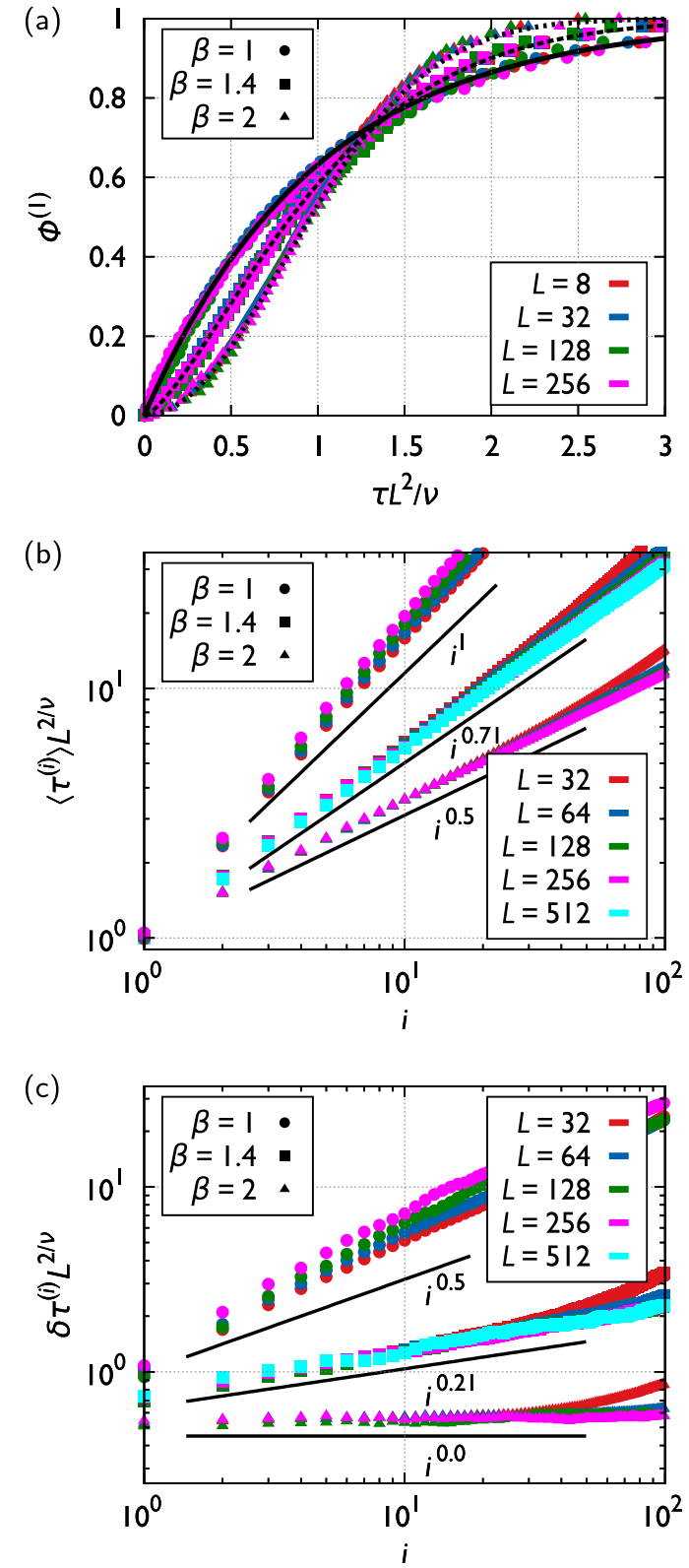

FIG. 5. (a) The cumulative distribution $\Phi^{(1)}$ of the first activation stress $\tau^{(1)}$ for SCPM simulations with $\beta=1,1.4,2$ and different system sizes. The scaling collapse for different system sizes is obtained by rescaling the stress by $L^{2 / \beta}$. The corresponding Weibull distributions of Eq. (16) are also plotted (solid, dashed, and dotted lines). (b) The average stress sequence $\left\langle\tau^{(i)}\right\rangle$. The mean initiation stress of the $i$ th event is proportional to $i^{1 / \beta}$ and initiation stresses corresponding to different system sizes collapse if stresses are rescaled by $L^{2 / \beta}$, in accordance with Eq. (9). (c) STD of the stress sequence $\delta \tau^{(i)}$. The curves are consistent with Eq. (10).

As for the SCPM, the average $\left\langle\tau^{(i)}\right\rangle$ and STD $\delta \tau^{(i)}$ follow Eqs. (9) and (10), respectively, with the same exponents $\beta$ and $\eta$ as obtained from $\Phi^{(1)}$ above.

\section{Strain sequence}

Figure 10 plots the average $\left(\left\langle\gamma^{(i)}\right\rangle\right)$ and STD $\left(\delta \gamma^{(i)}\right)$ of the strain sequence obtained for different system sizes. The
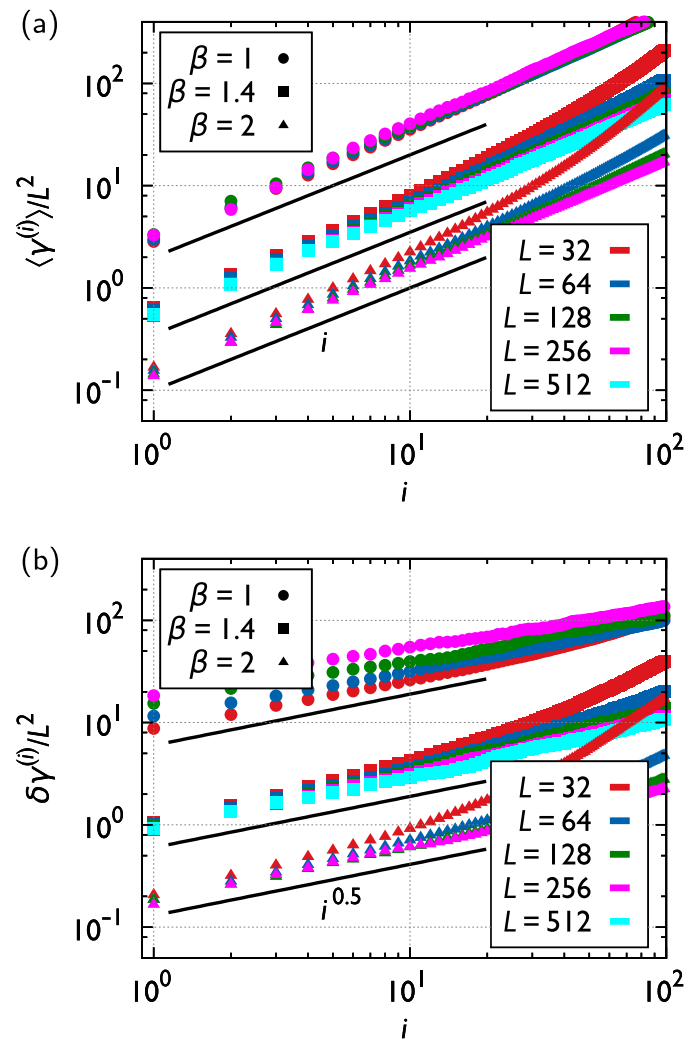

FIG. 6. The average [panel (a)] and STD [panel (b)] of the strain $\gamma^{(i)}$ measured at the $i$ th strain burst for different system sizes and $\beta$ values. The curves follow Eqs. (11) and (12) with $\zeta=1$ and $\xi=2$.

curves are consistent with Eqs. (11) and (12) found for the SCPM, with exponents $\zeta=0.9 \pm 0.05$ and $\xi=1.5 \pm 0.1 \mathrm{An}$ overview of the exponents we introduced to characterize the avalanche sequences, and of their values as deduced from the simulation data, is given in Table I.

\section{PLASTICITY MODEL BASED ON ORDER STATISTICS}

In this section a simple model for stochastic plasticity is introduced for stress-controlled loading. In this case the stress-strain curves are steplike and can be characterized by the stress and strain values $\tau^{(i)}$ and $\gamma^{(i)}$ corresponding to each step (see the sketch in Fig. 2). In the following, we propose a few simple assumptions for the initial stages of the stress and strain sequences and then combine them to obtain statistical predictions for the stress-strain curves. As we shall see, the proposed scaling forms will be identical to those obtained numerically in the previous section, so the same notation is used for the exponents (see Table I).

\section{A. Stress sequence}

Recently Derlet and Maaß proposed a probabilistic approach to explain size effects observed in crystalline specimens [33]. They assumed, in accordance with the main idea of the SCPM, that plasticity occurs via irreversible structural excitations and that the material inhomogeneities are represented by a critical stress distribution $P(\tau)=\frac{\mathrm{d}}{\mathrm{d} \tau} \Phi(\tau)$, with $\Phi(\tau)$ being the cumulative distribution. Since during stress increase the 

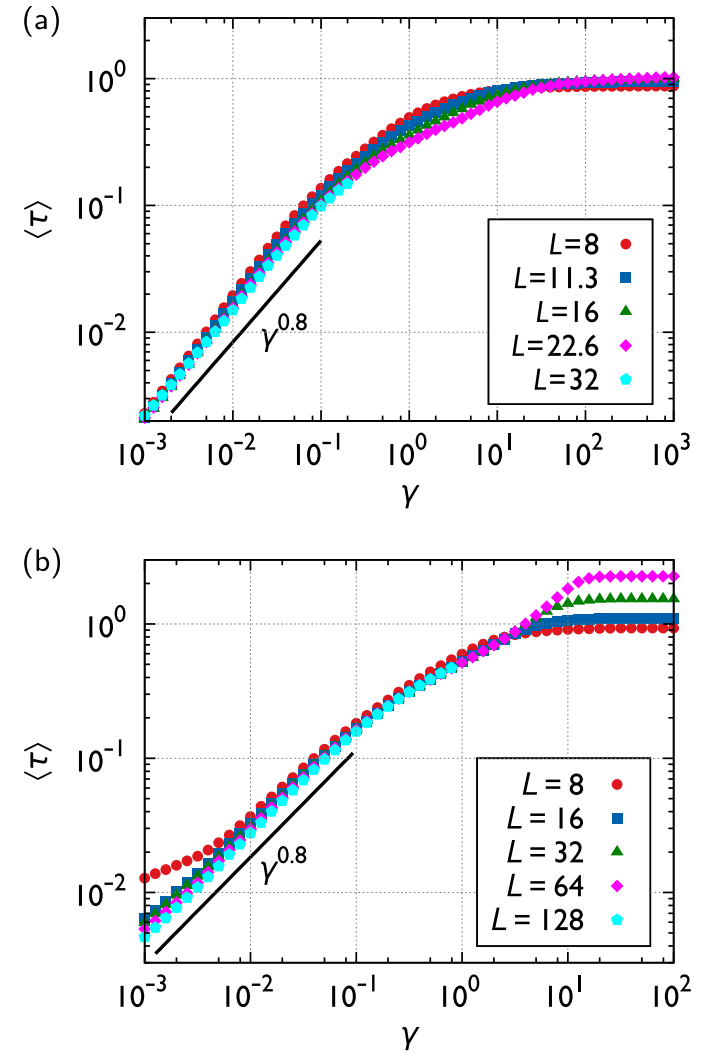

FIG. 7. The average stress-strain curves of the DDD simulations. They follow a power law until $\gamma \approx 0.1$, then saturate. The panels correspond to (a) TCDDD, (b) CADDD simulations.

weakest sites are activated, only the $\tau \rightarrow 0$ asymptote of this distribution is important, for which they used a power-law form

$$
\Phi(\tau) \approx\left(\frac{\tau}{\tau_{0}}\right)^{\beta}, \quad \text { if } \tau \rightarrow 0
$$

with $\beta \geqslant 1$. It was also assumed that the subsequent events are independent, so the spatial correlations of stress and plastic strain present in the SCPM were completely neglected. In this case, if the sample consists of $M$ sites where plastic events may initiate, then the $i$ th stress value $\tau^{(i)}$ in the $M \rightarrow \infty$ case follows Weibull order statistics [34]. In particular, the first event $\tau^{(1)}$ is Weibull distributed with shape parameter $\beta$ :

$$
\Phi^{(1, M)}\left(\tau^{(1, M)}\right)=1-\exp \left(-\frac{1}{M}\left(\frac{\tau^{(1, M)}}{\tau_{0}}\right)^{\beta}\right),
$$

the expected value for $\tau^{(i, M)}$ is

$$
\left\langle\tau^{(i, M)}\right\rangle=\frac{\tau_{0}}{M^{1 / \beta}} \frac{\Gamma\left(i+\frac{1}{\beta}\right)}{\Gamma(i)} \approx \tau_{0}\left(\frac{i}{M}\right)^{\frac{1}{\beta}},
$$

and for the standard deviation of $\tau^{(i, M)}$ one obtains

$$
\begin{aligned}
\delta \tau^{(i, M)} & =\sqrt{\left(\frac{\tau_{0}}{M^{1 / \beta}}\right)^{2}\left[\frac{\Gamma\left(i+\frac{2}{\beta}\right)}{\Gamma(i)}-\left(\frac{\Gamma\left(i+\frac{1}{\beta}\right)}{\Gamma(i)}\right)^{2}\right]} \\
& \approx \frac{\tau_{0}}{i^{1 / 2}}\left(\frac{i}{M}\right)^{\frac{1}{\beta}} .
\end{aligned}
$$

This means that the relative fluctuation decreases as $\delta \tau^{(i)} /\left\langle\tau^{(i)}\right\rangle \approx i^{-1 / 2}$, independent of the number of sites $M$. We also note that the error of these approximations is less than $2 \%$ if $i>5$ and $M \gg i$ (at $\beta=1.4$, see approximation 6.1 .47 in Ref. [35]).
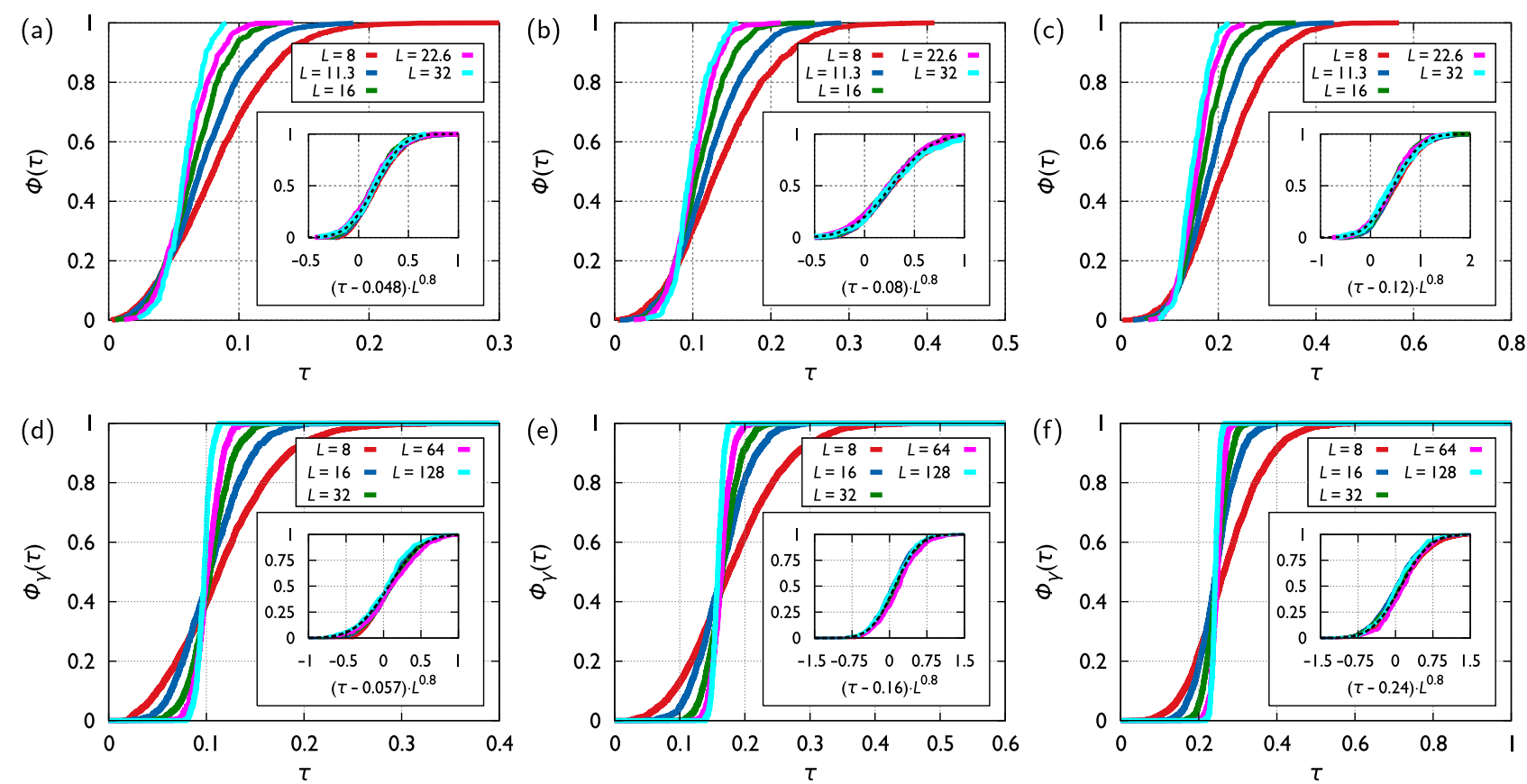

FIG. 8. Cumulative stress distributions $\Phi_{\gamma}$ at different deformation levels $\gamma$, DDD model. As in Fig. 4 scaling collapse can be obtained by multiplying the external stress with a power of the system size. The so collapsed curves can be fitted by an appropriate normal distribution (dashed lines). Panels (a)-(c) and (d)-(f) correspond to TCDDD and CADDD, respectively. (a), (d): $\gamma=0.05,(b),(e): \gamma=0.1,(c),(f): \gamma=0.2$. 


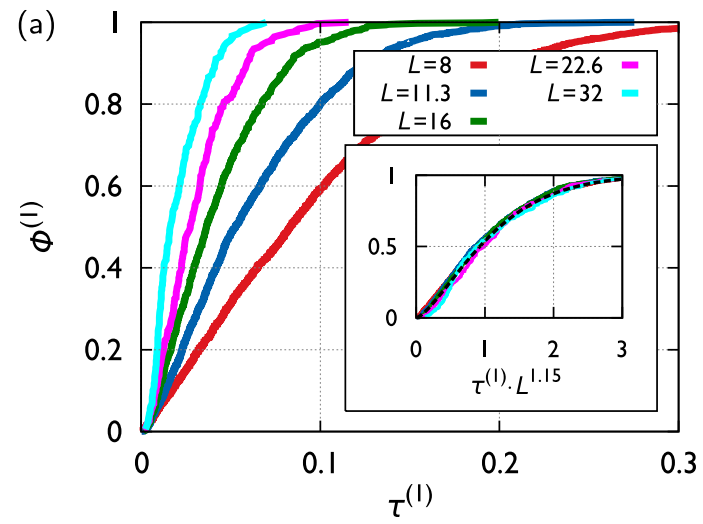

(b)

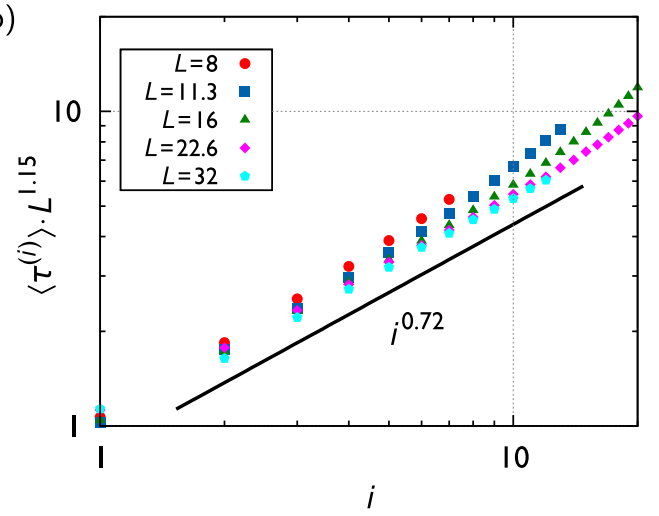

(c)

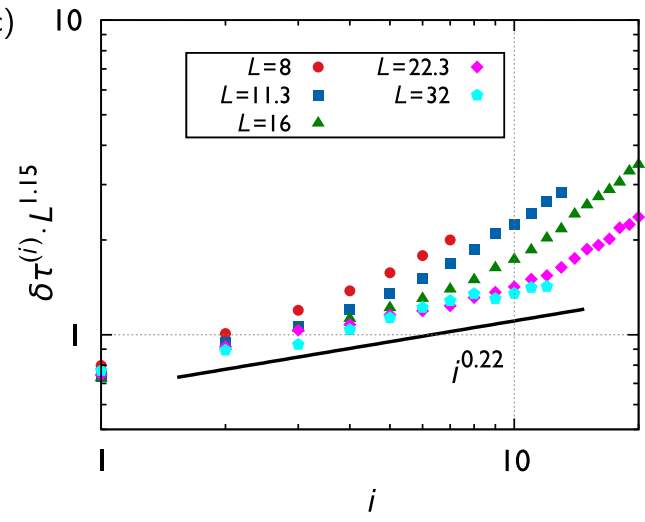

FIG. 9. Statistics of the stress sequence $\tau^{(i)}$ for TCDDD simulations of different system sizes.(a) Cumulative distribution $\Phi^{(1)}$ of the first activation stress $\tau^{(1)}$. Inset: data collapse obtained by plotting $\Phi^{(1)}$ as a function of $\tau L^{1.15}$. The curves can be fitted by a Weibull distribution with shape parameter $\beta \approx 1.4 \pm 0.05$. (b),(c) Average and STD of the external stress $\tau^{(i)}$ at the $i$ th avalanche for different system sizes. For sufficiently large system sizes, the data are consistent with Eqs. (9) and (10) (solid lines) with $\beta=1.4 \pm 0.05$ and $\eta=1.6 \pm 0.1$.

The above relations hold for $i \gg 1$ in the regime of extreme order statistics, that is if $M \rightarrow \infty$ such that $i / M \rightarrow 0$. Given that in this regime only a very small fraction of possible deformation events have actually occurred, one might wonder whether the use of extreme order statistics restricts the applicability of our considerations, in the large system limit, to negligible strains. To show that this is not the case, we demonstrate that the same relations carry over to the regime
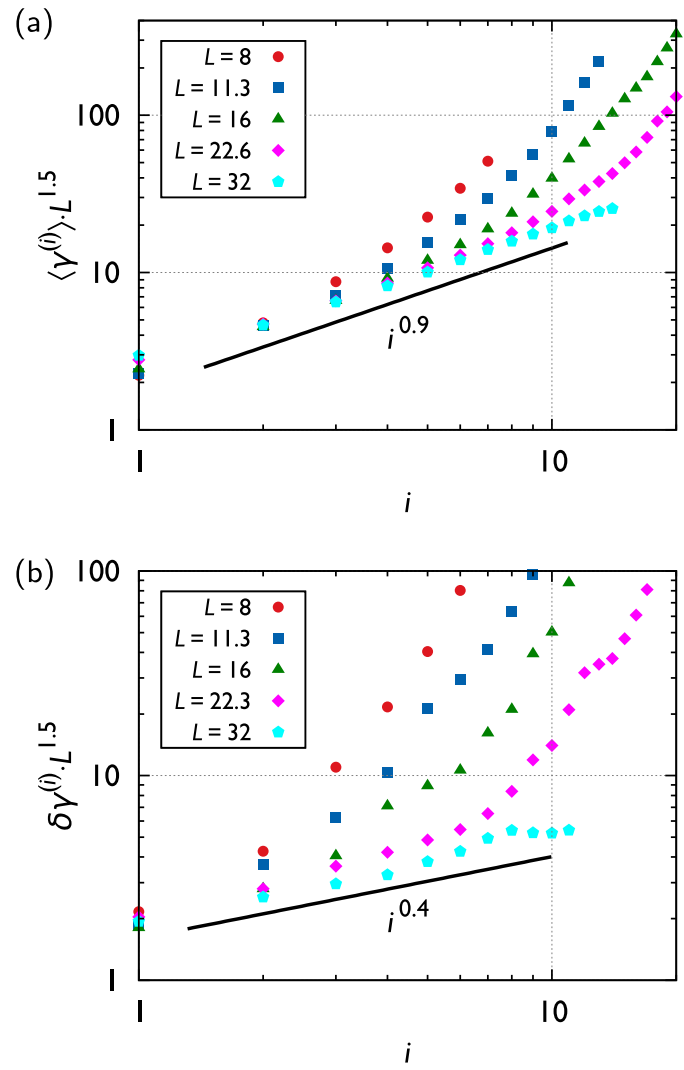

FIG. 10. Statistics of the strain sequence $\gamma^{(i)}$ for TCDDD simulations of different system sizes. The average [panel (a)] and STD [panel (b)] of the plastic strain $\gamma^{(i)}$ at the $i$ th avalanche for different system sizes. For sufficiently large system sizes, the data are consistent with Eqs. (11) and (12) (solid lines) with $\zeta=0.9 \pm 0.05$ and $\xi=1.5 \pm 0.1$

of central order statistics, that is, the regime where $i / M=p$ is finite.

In the asymptotic limit $M \rightarrow \infty, i / M=p$ finite, the distribution of the $i$ th member of an ordered list drawn from a sample of $M$ stresses with probability distribution $\Phi(\tau)$ is given by the normal distribution

$$
\Phi^{(i, M)}\left(\tau^{(i, M)}\right)=\frac{1}{2}\left[1+\operatorname{erf}\left(\frac{\tau^{(i, M)}-\left\langle\tau^{(i, M)}\right\rangle}{\sigma^{(i, M)}}\right)\right] .
$$

The mean and standard deviation are given by [36]

$$
\begin{aligned}
\left\langle\tau^{(i, M)}\right\rangle & =\Phi^{-1}(p)=: \tau(p), \\
\sigma^{(i, M)} & =\left(\frac{p(1-p)}{M\left[\Phi^{\prime}(\tau(p))\right]^{2}}\right)^{1 / 2},
\end{aligned}
$$

where $p=i / M, \Phi^{-1}$ is the inverse function (quantile function) of $\Phi$, and $\Phi^{\prime}=\partial \Phi / \partial \tau$. For the Weibull distribution in the power-law regime of small $p$ we find that $\Phi \approx\left(\tau / \tau_{w}\right)^{\beta}$ and it is easy to see that one recovers both Eqs. (17) and (18), hence, for the Weibull distribution the relations of extreme order statistics carry over into the regime of central order statistics.

Finally, one has to find the relation between $M$ and the linear system size $L$. It is natural to assume that the event initiation sites are homogeneously distributed and that their 
density does not depend on the sample size. This indicates $M \propto L^{d}$, with $d$ being the dimension of the system. As we shall see below this hypothesis must be refined for DDD systems due to anomalous system size scaling. Therefore, a generic exponent $\eta$ is introduced such that

$$
M \propto L^{\eta},
$$

leading to

$$
\begin{gathered}
\Phi^{(1)}\left(\tau^{(1)}\right)=1-\exp \left(-\left(\frac{1}{\tau_{0}} \frac{\tau^{(1)}}{L^{\eta / \beta}}\right)^{\beta}\right), \\
\left\langle\tau^{(i)}\right\rangle \approx \tau_{0} L^{-\eta / \beta} i^{1 / \beta}
\end{gathered}
$$

and

$$
\delta \tau^{(i)} \approx \tau_{0} L^{-\eta / \beta} i^{1 / \beta-1 / 2}
$$

\section{B. Strain sequence}

According to numerous recent experimental and numerical studies, the plastic strain increments, corresponding to the strain burst events, may exhibit power-law size distribution $[7,8,23,28]$. However, the scale-free behavior is observed only in a bounded region since (i) at large strain jumps the distribution is chopped off either due to finite system size or due to intrinsic bounds inherent in the dynamics, and (ii) in the case of very small strain bursts, deviation from the power law is necessary otherwise the strain burst distribution could not be normalized. The physical origin of this lower cutoff is that here individual dislocation motion dominates over collective dislocation dynamics. In summary, the strain burst size $(\Delta \gamma)$ distribution is given by

$$
P_{\mathrm{sb}}(\Delta \gamma)=C \Delta \gamma^{-\tau_{a}} f\left(\Delta \gamma / \Delta \gamma_{u}\right) \text {, if } \Delta \gamma>\Delta \gamma_{l},
$$

where $\Delta \gamma_{l}$ and $\Delta \gamma_{u}$ represent lower and upper cutoffs, respectively, $\tau_{a}$ is the avalanche size exponent, $C$ is a normalization factor, and $f$ is a cutoff function with $f(x) \rightarrow 1$ for $x \rightarrow 0$ that decays faster than algebraically for large arguments. This scaling form includes the possibility $\tau_{a}=0$ when no power-law regime can be identified.

It follows that for finite system sizes and small applied stresses, due to the cutoffs, the distribution $P_{\mathrm{sb}}(\Delta \gamma)$ has finite moments - in particular, finite mean and variance. The recent numerical study of 2D DDD systems of Ispánovity et al. showed that in the microplastic regime the exponent $\tau_{a} \approx 1$, the upper cutoff $\Delta \gamma_{u}$ depends weakly on the applied stress, and it exhibits anomalous system size dependence [28]. In the SCPM, by contrast, recent investigations indicate that $\tau_{a} \approx 1.35$, the upper cutoff $\Delta \gamma_{u}$ diverges at a critical stress, and it exhibits normal system size dependence [20]. Despite these differences, in the microplastic regime the mean and variance of the strain increment can in both cases be written as

$$
\begin{gathered}
\langle\Delta \gamma\rangle=\frac{s_{0}}{L^{\xi}}, \\
\delta(\Delta \gamma)=\frac{s_{1}}{L^{\xi}},
\end{gathered}
$$

respectively, where $\xi$ is the exponent characterizing the system size dependence of the avalanche sizes, and $s_{0}$ and $s_{1}$ are appropriate constants. Here $\xi=2$ corresponds to normal scaling, where the total plastic slip during an avalanche is independent of the system size, whereas $\xi<2$ indicates anomalous scaling.

In order to derive predictions for the strain sequence it is assumed that the sizes of subsequent strain bursts are uncorrelated. Then from the central limit theorem it follows that for $i \gg 1$ and small applied stresses, $\gamma^{(i)}$ is distributed normally, and

$$
\begin{gathered}
\left\langle\gamma^{(i)}\right\rangle=i^{\zeta}\langle\Delta \gamma\rangle=s_{0} \frac{i^{\zeta}}{L^{\xi}}, \\
\delta \gamma^{(i)}=i^{\zeta-1 / 2} \delta(\Delta \gamma)=\frac{s_{1}}{i^{1 / 2}} \frac{i^{\zeta}}{L^{\xi}},
\end{gathered}
$$

with $\zeta=1$.

\section{Stress-strain curves}

Since the sequences $\tau^{(i)}$ and $\gamma^{(i)}$ give a full description of the stress-strain curve, in the following the expressions for $\tau^{(i)}$ and $\gamma^{(i)}$ derived above are combined to obtain statistical properties of the stress-strain curves. It was predicted that for $i \gg 1$ both $\gamma^{(i)}$ and $\tau^{(i)}$ are Gaussian distributed [see Eqs. (28),(29) and Eqs. (17),(18)]. By "inverting" $\gamma^{(i)}$ to express $i$ at a given plastic strain $\gamma$, and then inserting $i(\gamma)$ into $\tau^{(i)}$ one obtains that, for $i \gg 1, \tau$ is Gaussian distributed and

$$
\begin{gathered}
\langle\tau\rangle=\frac{\tau_{0}}{s_{0}^{1 / \beta \zeta}} L^{(\xi / \zeta-\eta) / \beta} \gamma^{1 / \beta \zeta}, \\
\delta \tau=\tau_{2} L^{(\xi / \zeta-\eta) / \beta-\xi / 2 \zeta} \gamma^{1 / \beta \zeta-1 / 2 \zeta} .
\end{gathered}
$$

This means that the average stress-strain curve starts as a power law and if $\xi / \zeta \neq \eta$ then it has a system size dependence even at very large system sizes. To exclude this nonphysical situation one requires in the large-system limit that

$$
\xi=\eta \zeta .
$$

In this case the average and the fluctuation of the stress-strain curve behave as

$$
\begin{gathered}
\langle\tau\rangle \propto \gamma^{\alpha}, \\
\delta \tau \propto L^{-\theta},
\end{gathered}
$$

with

$$
\begin{gathered}
\alpha=1 / \beta \zeta, \\
\theta=\eta / 2 .
\end{gathered}
$$

Since $\eta$ is positive, this means that stress fluctuations decrease as size increases, that is, one obtains a smooth stress-strain curve for very large samples, as expected.

To summarize this section, a weakest link assumption proposed by Derlet and Maaß was adopted for the stress sequence [Eqs. (17),(18)] [33], and a straightforward rule for the strain sequence was proposed which is able to capture the anomalous system size dependence of 2D DDD systems [Eqs. (28),(29)] [28]. The combination of the two series has led to statistical predictions on the stress-strain curves [Eqs. (30),(31)], which, in fact, coincide with the numerical findings described in Sec. IV. In addition, the scaling relations 
Eqs. (32) and (35),(36) also hold for the numerically obtained data (see Table I).

The whole argument is based upon the assumptions that the Weibull distribution of the avalanche thresholds remains unchanged by the previous avalanches, that the avalanche size statistics is stress independent, and that there are many avalanches taking place over the considered stress and strain range such that the sum of their strains is Gaussian distributed according to the central limit theorem. The latter can be always ensured by considering sufficiently large systems. However, the first two conditions may be difficult to reconcile with a divergence of the avalanche size which is, in the SCPM model, observed at a critical stress. They are however valid in the regime of small stresses where avalanches are essentially localized, rather than system-spanning events.

\section{DISCUSSION}

In the preceding two sections numerical results and a theory were presented yielding identical scaling forms for the average and fluctuation of the individual stress-strain curves and the stress/strain sequence in the microplastic regime. The exponents introduced to describe these quantities and their measured/predicted values are summarized in Table I. In the following discussion we highlight the most important consequences of these findings.

Firstly, we consider the main idea of SCPM that the material can be decomposed into local units each characterized by a yield threshold accounting for the inhomogeneity of the underlying microstructure (e.g., dislocation patterns). This nontrivial assumption implies that the distribution of $\tau^{(1)}$, i.e., the stress at the onset of the first plastic event, must follow a weakest link distribution. So, the fact that for DDD a Weibull distribution was found to describe $P\left(\tau^{(1)}\right)$ supports this fundamental hypothesis. In addition, it gives us access to the distribution of local strengths of pinned or jammed configurations in dislocation structures, since the shape parameter $\beta$ unambiguously determines the asymptote of the underlying strength distribution, in this case a power law, cf. Eq. (15). As such, the exponent $\beta$ emerges as a central parameter that also influences the power-law exponent of the plastic stress-strain relation $(\alpha=1 / \beta \zeta)$, that is, the amount of plasticity in the microplastic regime (see Fig. 3). The origin of $\beta \approx 1.4$ for DDD systems is not addressed in this paper, it may be influenced by the internal structure of dislocation arrangements, like slip systems, patterns, etc. It is noted, however, that a similar analysis of the average stress-strain curves performed earlier on 3D DDD simulations and micropillar compressions yielded $\alpha \approx 0.8$ in both cases [24,32], hinting at some generality in the value of $\beta$ (with the straightforward assumption of $\zeta=1$ ). We note that according to recent investigations of Tüzes $e$ al. the parameter $\beta$ plays a central role in the ductility of a system exhibiting strain softening [37].

Although the behavior of the stress sequence shows strong similarity between DDD and SCPM, the exponent $\eta$ characterizing the system size dependence of the number of "links" of the system $M$ differs considerably. For SCPM $\eta \approx 2$ was found, corresponding to a proportionality between $M$ and the 2D system size, whereas for DDD a significantly smaller

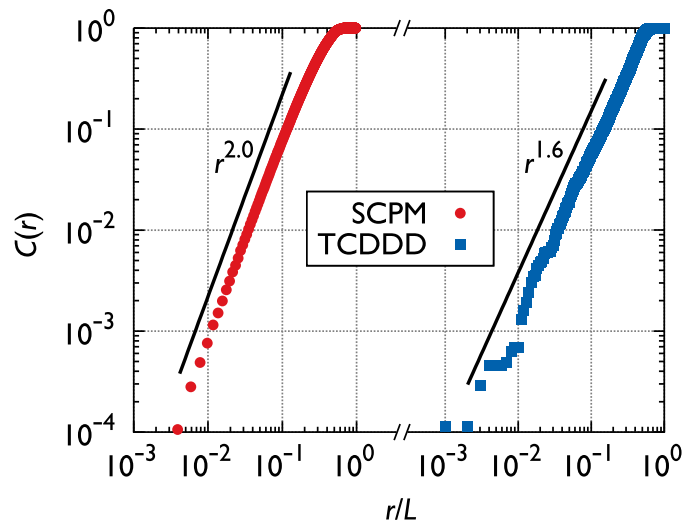

FIG. 11. Correlation integral of the avalanche positions for the SCPM and TCDDD models. The measured data are consistent with $C(r) \propto r^{\eta}$, the latter indicated by the solid lines.

value of $\eta \approx 1.5$ was obtained, hinting at a fractal-like structure of the weakest regions of the system. In order to quantify this conjecture we consider the correlation integral $C(r)$ of the initiation points of the events, defined as the probability of the distance of two such arbitrarily chosen points being smaller than $r$. A fractal dimension $d$ can be defined from the asymptotic behavior as $C(r) \propto r^{d}$. Indeed, according to Fig. 11 the $C(r) \propto r^{\eta}$ is a very good approximation both for SCPM and DDD. Although the explanation of this difference is beyond the scope of the present paper we mention that the fact that plasticity accumulates on a fractal-like subdomain of the system may explain the recent findings on the long-range nature of dynamical correlations and peculiar critical behavior of 2D DDD systems [28]. In addition, it echoes on the experimental findings of Weiss et al., where it was found that during creep deformation of an ice single crystal acoustic emission signals initiate from a fractal subvolume of the specimen with dimension $\sim 2.5$ [38].

Although we discuss many aspects of system size dependence throughout the paper we would like to clarify that these effects are different from the size effects observed in a range of materials, generally described as "smaller is stronger." Such effects may stem from different sources related to boundary effects-in systems with constrained boundaries, dislocations may pile up at boundaries and cause significant, size dependent back stresses, whereas in systems with open boundaries, strengthening may conversely be caused by loss of dislocations at the surface (exhaustion hardening). Since our focus here was on bulk properties we avoid both types of effects by implementing periodic boundary conditions for all models. These boundary conditions do not lead to these type of size effects as evident from Figs. 3(a) and 7 and can, hence, be taken to adequately represent bulk behavior (N.B.: Fig. 7 shows an inverse size effect at large strains. This effect is related to the periodic wrapping of slip planes onto themselves by the periodic BC and must be deemed unphysical, as discussed in Ref. [27]).

Despite the differences in the system size scaling of the fluctuations between SCPM and DDD models described above, the presented theoretical model gives a close approximation to describe the microplastic regime in both cases. According to the scaling relations derived [Eqs. (32) and (35),(36)] and 


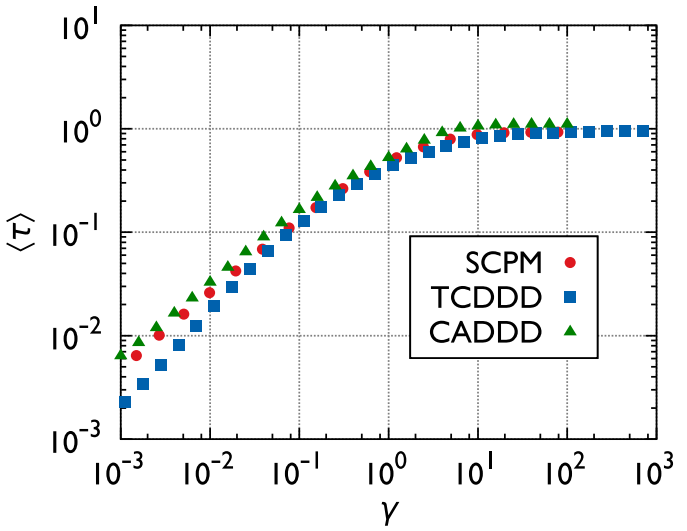

FIG. 12. Stress-strain curves obtained by the three different plasticity models. For the SCPM $\beta=1.4, \Delta \gamma_{\mathrm{pl}}=6$, and $\tau_{w}=2$ was chosen.

making the straightforward $\zeta=1$ assumption (see Sec. V B) there are only two independent exponents left, namely $\beta$ and $\eta$. Although these parameters represent microstructural details of the material (local strength distribution and fractal dimension of the initiation sites, respectively) they are directly linked to macroscopically measurable quantities: (i) $\beta$ is related to the power-law exponent characterizing the stress-strain curve in the microplastic regime while $(\eta)$ can be determined from the stress fluctuations of different samples (see Table I). Therefore, the model parameters can be determined both from lower and larger scale models, even from experiments. According to Fig. 12 the SCPM configured in this manner properly describes the microplastic regime. Interestingly, with a proper choice of the parameters the average stress-strain curves overlap for large strains, too, but since SCPM does not properly describe internal strain correlations developing at large deformations [27] this is not expected to bear physical relevance.

\section{SUMMARY}

In this paper we have demonstrated that the SCPM model introduced earlier is able to quantitatively describe the stochastic properties of crystalline plasticity in the microplastic regime. Using a simple theoretical model based on the subsequent activation of the weakest links in the sample we derived a method how to calibrate the parameters of the SCPM based on lower-level DDD simulations. The proposed methodology does not only represent a bridge between micro- and mesoscales, but also gives insight into the nature of the stochastic processes characterizing plasticity. The current paper has focused on crystal plasticity and a simple 2D DDD representation, but the authors do not see any reason why the proposed plasticity model and the multiscale methodology would not be applicable for more involved DDD models or amorphous materials, too. The verification of this conjecture is relegated to future work and is expected to open new perspectives in the applicability of stochastic continuum plasticity models.

\section{ACKNOWLEDGMENTS}

P.D.I. thanks Peter Derlet for fruitful discussions. Financial support of the Hungarian Scientific Research Fund (OTKA) under Contracts No. K-105335 and No. PD-105256 and of the European Commission under Grant Agreement No. CIG321842 are also acknowledged. P.D.I. is also supported by the János Bolyai Scholarship of the Hungarian Academy of Sciences. M.Z. acknowledges financial support by DFG under Project No. Za 171/7-1.
[1] I. Groma, F. Csikor, and M. Zaiser, Acta Mater. 51, 1271 (2003).

[2] I. Groma, Z. Vandrus, and P. D. Ispánovity, Phys. Rev. Lett. 114, 015503 (2015).

[3] T. Hochrainer, S. Sandfeld, M. Zaiser, and P. Gumbsch, J. Mech. Phys. Solids 63, 167 (2014).

[4] L. H. Poh, R. Peerlings, M. Geers, and S. Swaddiwudhipong, J. Mech. Phys. Solids 61, 913 (2013).

[5] V. Levkovitch and B. Svendsen, Int. J. Solids Struct. 43, 7246 (2006).

[6] R. Sedláček, C. Schwarz, J. Kratochvil, and E. Werner, Philos. Mag. 87, 1225 (2007).

[7] D. M. Dimiduk, C. Woodward, R. LeSar, and M. D. Uchic, Science 312, 1188 (2006).

[8] M. Zaiser, J. Schwerdtfeger, A. Schneider, C. Frick, B. G. Clark, P. Gruber, and E. Arzt, Philos. Mag. 88, 3861 (2008).

[9] F. F. Csikor, C. Motz, D. Weygand, M. Zaiser, and S. Zapperi, Science 318, 251 (2007).

[10] M. D. Uchic, Science 305, 986 (2004).

[11] D. Dimiduk, M. Uchic, and T. Parthasarathy, Acta Mater. 53, 4065 (2005).

[12] M. D. Uchic, P. A. Shade, and D. M. Dimiduk, Annu. Rev. Mater. Res. 39, 361 (2009).
[13] M. Zaiser and P. Moretti, J. Stat. Mech.: Theor. Exp. (2005) P08004.

[14] M. Zaiser and N. Nikitas, J. Stat. Mech.: Theor. Exp. (2007) P04013.

[15] S. Papanikolaou, D. M. Dimiduk, W. Choi, J. P. Sethna, M. D. Uchic, C. F. Woodward, and S. Zapperi, Nature (London) 490, 517 (2012).

[16] V. Bulatov and A. Argon, Modell. Simul. Mater. Sci. Eng. 2, 167 (1994).

[17] J.-C. Baret, D. Vandembroucq, and S. Roux, Phys. Rev. Lett. 89, 195506 (2002).

[18] M. Talamali, V. Petäjä, D. Vandembroucq, and S. Roux, Phys. Rev. E 84, 016115 (2011).

[19] Z. Budrikis and S. Zapperi, Phys. Rev. E 88, 062403 (2013).

[20] S. Sandfeld, Z. Budrikis, S. Zapperi, and D. F. Castellanos, J. Stat. Mech.: Theor. Exp. (2015) P02011.

[21] C. Liu, E. E. Ferrero, F. Puosi, J.-L. Barrat, and K. Martens, Phys. Rev. Lett. 116, 065501 (2016).

[22] J. D. Eshelby, Proc. R. Soc. London A 241, 376 (1957).

[23] M.-C. Miguel, A. Vespignani, S. Zapperi, J. Weiss, and J.-R. Grasso, Nature (London) 410, 667 (2001). 
[24] P. D. Ispánovity, I. Groma, G. Györgyi, F. F. Csikor, and D. Weygand, Phys. Rev. Lett. 105, 085503 (2010).

[25] G. Tsekenis, N. Goldenfeld, and K. A. Dahmen, Phys. Rev. Lett. 106, 105501 (2011).

[26] M. Ovaska, L. Laurson, and M. J. Alava, Sci. Rep. 5, 10580 (2015).

[27] O. Kapetanou, D. Weygand, and M. Zaiser, J. Stat. Mech.: Theor. Exp. (2015) P08009.

[28] P. D. Ispánovity, L. Laurson, M. Zaiser, I. Groma, S. Zapperi, and M. J. Alava, Phys. Rev. Lett. 112, 235501 (2014).

[29] M. Zaiser and S. Sandfeld, Modell. Simul. Mater. Sci. Eng. 22, 065012 (2014).

[30] B. Bako, I. Groma, G. Györgyi, and G. Zimanyi, Comput. Mater. Sci. 38, 22 (2006).
[31] P. Szabó, P. D. Ispánovity, and I. Groma, Phys. Rev. B 91, 054106 (2015).

[32] P. D. Ispánovity, A. Hegyi, I. Groma, G. Györgyi, K. Ratter, and D. Weygand, Acta Mater. 61, 6234 (2013).

[33] P. M. Derlet and R. Maass, Philos. Mag. 95, 1829 (2015).

[34] H. Rinne, The Weibull Distribution: A Handbook (CRC Press, Boca Raton, FL, 2008).

[35] M. Abramowitz and I. Stegun (eds.), Handbook of Mathematical Functions, 9th ed. (Dover, New York, 1970), p. 257.

[36] A. DasGupta, Asymptotic Theory of Statistics and Probability (Springer, New York, NY, 2008).

[37] D. Tüzes, P. D. Ispánovity, and M. Zaiser, arXiv:1604.01821 [Int. J. Fract. (to be published)].

[38] J. Weiss and D. Marsan, Science 299, 89 (2003). 